\title{
Characterization of Breast Lesions: Comparison between Three-dimensional Ultrasound and Automated Volume Breast Ultrasound
}

Wanru Jia, MD ${ }^{a, 1}$, Jingwen, Zhang, MD ${ }^{a, 1}$, Yijie Dong, MD ${ }^{a}$, Ying Zhu, MD ${ }^{a}$, Xiaohong Jia, MD ${ }^{a}$, Weiwei Zhan, $M^{a}$, Jianqiao Zhou, MD ${ }^{a, *}$

${ }^{a}$ Ruijin Hospital, School of Medicine, Shanghai Jiaotong University, Shanghai, China

Received, March 8, 2021; revision received April 10, 2021; accepted April 15, 2021

Objective: This study aimed to compare the diagnostic performance of three-dimensional ultrasound (3D-US) and automated breast volume scanner (ABVS) for the characterization of benign and malignant breast lesions.

Methods: Ninety patients who underwent surgery and preoperative conventional ultrasound (US), 3D-US, and ABVS examinations were enrolled in this study. The image quality and adjacent structures of the lesions in the coronal plane were compared. The combination of US, 3D-US, and ABVS for retraction phenomenon of the lesion was compared and the diagnostic performance of each combination was analyzed.

Results: ABVS displayed better image quality and adjacent structures than 3D-US $(P<0.001)$. The area under the curve (AUC) was $0.913,0.842$, and 0.871 for US, 3D-US, and ABVS, respectively. The AUC of the retraction phenomenon of the lesion was 0.732 and 0.810 for 3D-US and ABVS, respectively. When they were combined, US+ABVS showed the highest AUC of 0.924. No significant difference of diagnostic performances was found among conventional US, US $+3 \mathrm{D}-\mathrm{US}$, and US + ABVS $(P>0.05)$.

Conclusions: Compared with 3D-US, ABVS seems to be superior in showing the retraction phenomenon of breast lesions and in the characterization of breast lesions alone or in combination with conventional US. Although no significant difference was observed between them, both ABVS and 3D-US provided valuable information in the coronal plane and improved our confidence level in breast lesion characterization, especially when combined with the conventional US.

Key words: Breast neoplasms; Ultrasonography; Imaging; Three-dimensional; Automated volume scanner

Advanced Ultrasound in Diagnosis and Therapy 2021; 03: 204-211

DOI: 10.37015/AUDT.2021.210007

$\mathrm{M}$ ammography is currently the primary screening and diagnostic tool for the detection of breast cancer. However, the sensitivity of mammography is substantially low for women with high breast density [1, 2]. A study carried out by Mandelson et al. was noted that the mammographic sensitivity was only $30 \%$ among women with extremely dense breasts but $80 \%$ in women with predominantly fatty breasts [3].
Moreover, women with high density breast have been shown to have an increased risk of developing breast cancer [4]. Therefore, additional imaging techniques, including ultrasound (US) and magnetic resonance imaging (MRI), have been used as a supplement to mammography for the detection of malignancy [5].

For women with dense breast tissue, mammographically occult or subtle cancers can be identified by screening

\footnotetext{
${ }^{1}$ These authors contributed equally to this work and to be considered as co-first authors.

" Corresponding author: Department of Ultrasound, Ruijin Hospital, Shanghai Jiaotong University School of Medicine, No.197 Rui Jin 2nd Road, Shanghai 200025, China

e-mail: zhousu30@126.com
} unrestricted use, distribution and reproduction in any medium provided that the original work is properly attributed. 
with combined US and mammography [1,6,7]. A study with a larger sample size has revealed that the US can detect more breast cancers at a lower stage or with a smaller size compared with physical examination [8]. However, the US is much more operator-dependent than other imaging modalities with a significant learning curve, and the image quality of the US is strongly related to the operator's expertise and skill.

In recent years, three-dimensional US (3D-US) has emerged to provide a new perspective in breast ultrasound [9-11], which could reduce operator dependence for volumetric images of the breast to be acquired in a standardized manner. Additionally, the anatomical planes of interest within the volume can be visualized and reviewed in multiplanar views, including the transverse, sagittal, and coronal planes [12-14]. In the last few years, an automated whole breast ultrasound equipment, i.e., automated breast volume scanner (ABVS), has been increasingly used in breast imaging practice, including detection and characterization [11,15-17], and assessment of treatment response after neoadjuvant chemotherapy [18]. Compared with conventional 3D-US, the advantage of ABVS lies in a large field of view, so that the breast can be fully displayed by only two to four-volume scans $[11,15,19]$.

Many studies have compared handheld two-dimensional US and 3D-US for diagnostic performance in the detection and characterization of breast lesions [10,20-22], and several studies have compared handheld twodimensional US with ABVS from different perspectives [15,23-26]. However, no studies have compared the diagnostic accuracy of ABVS to 3D-US in terms of breast lesion characterization.

Thus, the purpose of our study was to compare the diagnostic performance of ABVS and 3D-US in differentiating benign from malignant breast lesions with histopathologic examination as the reference standard.

\section{Materials and Methods}

\section{Patients}

This prospective study was approved by our institutional ethics committee, and informed consent was obtained from all patients at the time of examination. From November 2015 to December 2016, 96 consecutive women who underwent preoperative conventional US, 3D-US, and ABVS examinations in our department were enrolled in this study. The indications for the US were abnormal findings on breast imaging, palpable masses, and nipple discharge. Eight women were later excluded from the study for the following reasons: 3 patients without histopathological confirmation, 2 patients with a history of surgical intervention, and 1 patient without complete US data. Finally, 90 breast lesions in 90 patients were included in the final data analysis.

\section{Conventional US and 3D-US examination}

Conventional US and 3D-US examinations were performed by two radiologists (J.Q.Z. and W.W.Z.) with more than 20 years of experience in breast US. Conventional US examinations were performed using a MyLab 90 scanner (Esaote SpA, Genoa, Italy) equipped with a LA523 (4-13 MHz) multiple-frequency linear array transducer while 3D-US images were obtained using same scanner with a BL433 (4-13 MHz) volumetric multiple-frequency linear array transducer. The footprint of the BL433 transducer was $55 \times 28 \mathrm{~mm}$. When acquiring $3 \mathrm{D}$ volume data, the field of view was set to $44 \mathrm{~mm}$ (maximum), and the scan angle was set to $50^{\circ}$ (maximum). The scan plane was mechanically moved by a build-in motor controlled by the scanner system, allowing a uniform volume reconstruction based on the volume acquisition technology developed by Esaote.

During 3D-US scanning, the probe was held still, and the patients were asked to hold their breath for approximately 5 seconds. Subsequently, three perpendicular planes, i.e., sagittal, transverse, and coronal planes, of the target region were displayed simultaneously on the scanner monitor. The acquired 3D volume data and representative sagittal, transverse, and coronal images were then stored on the system hard drive for document and analysis.

\section{ABVS examination}

Following the conventional US and 3D-US, ABVS examination was performed using the Acuson S2000 ultrasound unit (Siemens Medical Solutions, Mountain View, CA, USA), combined with 6-14 MHz broadband mechanical transducers. During the examination, the patient was placed in a supine position with both breasts fully exposed. The transducer was covered with a replaceable membrane to allow for adequate contact with the skin. The anterior, posterior, lateral, and medial views were acquired for each breast, and additional views were needed for larger breasts. On average, the total time to complete the examination was $15 \mathrm{~min}$, including patient preparation, probe positioning, and scanning. Once the images were obtained, they were sent to a dedicated workstation for the multiplanar reconstruction of the images, including the transverse, sagittal, and coronal planes.

\section{Image analysis}

All images, including conventional US, 3D-US, and ABVS, were reviewed by two radiologists (Y.Z and Y.J.D) with more than 10 years of experience in breast US without prior knowledge of the patients' histories, pathological findings, or other imaging results. Based 
on the fifth edition of the BI-RADS lexicon for breast ultrasound [27], all characteristics for each technique were reviewed as follows: shape (oval, round, or irregular), orientation (parallel or not parallel), margin (circumscribed, indistinct, micro lobulated, angular, or spiculated), echo pattern (hypoechoic, hyperechoic, isoechoic, anechoic, complex cystic and solid, or heterogeneous), posterior features (no posterior features, enhancement, shadowing, or combined pattern), calcification (presence or absence), and associated features (architectural distortion, ductal changes, skin changes, or edema), and a final BI-RADS category was given for US, 3D-US, and ABVS. A consensus would be obtained if there were any controversies by two readers. The retraction phenomenon reflecting malignant characteristics in the coronal plane was defined as hyperechoic cordlike structures interspersed with hypoechoic interval converging into part or all the lesion periphery [28], which was assessed and interpreted for $3 \mathrm{D}-\mathrm{US}$ and ABVS images. The retraction phenomenon of 3D-US and ABVS was respectively combined with the conventional US for modified BI-RADS categories. If the retraction existed in conventional US, the BIRADS categories would upgrade. However, those with BI-RADS 5 could not be upgrade anymore; thus, the receiver operating curve (ROC) of modified BI-RADS categories would obtained and compared.

In addition, the image quality and display of adjacent structures of the lesion in the coronal plane, which can reflect the overall spatial structure of breast lesions, were compared by two radiologists to determine which one was better. The judgment was based on the comprehensive resolution and contrast information of each image. If there was disagreement, a consensus would be reached after discussion.

\section{Pathological analysis}

All patients underwent core needle biopsy or surgery within one week after US examination. The final histopathological diagnosis was made by one pathologist with 25 years of experience in breast diseases, and blinded to all imaging results.

\section{Statistical analysis}

SPSS (version 21.0; SPSS Inc., Chicago, IL, USA) was used for general statistical analysis and MedCalc 19.05 (MedCalc Software, Mariakerke, Belgium) for ROC analysis using the histological results as the gold standard. The Wilcoxon signed-rank test was used to compare the image quality and display of adjacent structures of the lesion between 3D-US and ABVS. The sensitivity, specificity, area under the curve (AUC), and cut-off value of the BI-RADS category were calculated and compared among conventional US, 3D-US, and ABVS, respectively. The BI-RADS categories were dichotomized using $>4 \mathrm{~A}$ as the cutoff value, and the Kappa statistic was calculated for consistency check between any two imaging techniques. $P<0.05$ was considered statistically significant, and the kappa value was characterized as follows: $<0.4$ as slight, $0.4-0.75$ as moderate, and $\geqslant 0.75$ as good agreement.

\section{Results}

\section{Pathological results}

The mean age of all 90 patients was $46.93 \pm 13.85$ (range 18-78) years old, and the mean diameters of all breast lesions were $20.3 \pm 10.2 \mathrm{~mm}$ (range, 5.7 - 50.9 $\mathrm{mm}$ ). Additionally, the pathological results were listed in Table 1. Among the 55 benign breast lesions, 35 cases were fibroadenoma $(38.89 \%)$, 9 cases were adenosis $(10 \%)$, and 7 cases were intraductal papilloma (7.78\%); while there were 35 malignant lesions, including 27 cases of invasive ductal carcinoma (30\%), 4 cases of ductal carcinoma in situ $(4.44 \%)$, one case of invasive lobular carcinoma $(1.11 \%)$, one case of mucinous carcinoma $(1.11 \%)$, one case of metaplastic carcinoma $(1.11 \%)$, and one case of solid neuroendocrine carcinoma $(1.11 \%)$.

Table 1 Pathologic results of all patients

\begin{tabular}{|c|c|}
\hline Item & Cases \\
\hline Benign & 55 \\
\hline Fibroadenoma & 35 \\
\hline Adenosis & 9 \\
\hline phyllode tumor & 2 \\
\hline Papillary ductal carcinoma & 7 \\
\hline Pseudoangiomatous hyperplasia & 1 \\
\hline Ductal dilation with cyst & 1 \\
\hline Malignant & 35 \\
\hline Invasive ductal carcinoma & 27 \\
\hline Invasive lobular carcinoma & 1 \\
\hline Ductal carcinoma in situ & 4 \\
\hline Mucinous carcinoma & 1 \\
\hline Metaplastic carcinoma & 1 \\
\hline Solid neuroendocrine carcinoma & 1 \\
\hline Total & 90 \\
\hline
\end{tabular}




\section{Comparison of overall image quality between $3 D-U S$ and $A B V S$}

Table 2 illustrated the overall image quality of 3DUS and ABVS. In terms of image quality, there was no significant difference between 3D-US and ABVS $(P=$ 0.330). However, for the display of adjacent structures, ABVS was superior to 3D-US with statistical significance $(P<0.001)$. In 51 of the 90 cases, ABVS displayed better image quality of the adjacent structures than $3 \mathrm{D}$-US (Fig. 1-3).

\begin{tabular}{lcc} 
Table 2 Overall image quality of 3D-US and ABVS \\
\hline Item & Image quality & Display of adjacent structures \\
\hline 3D-US better & 16 & 4 \\
ABVS better & 22 & 51 \\
Similar & 52 & 35 \\
\hline
\end{tabular}

3D-US, three-dimensional ultrasound; ABVS, automated breast volume scanner
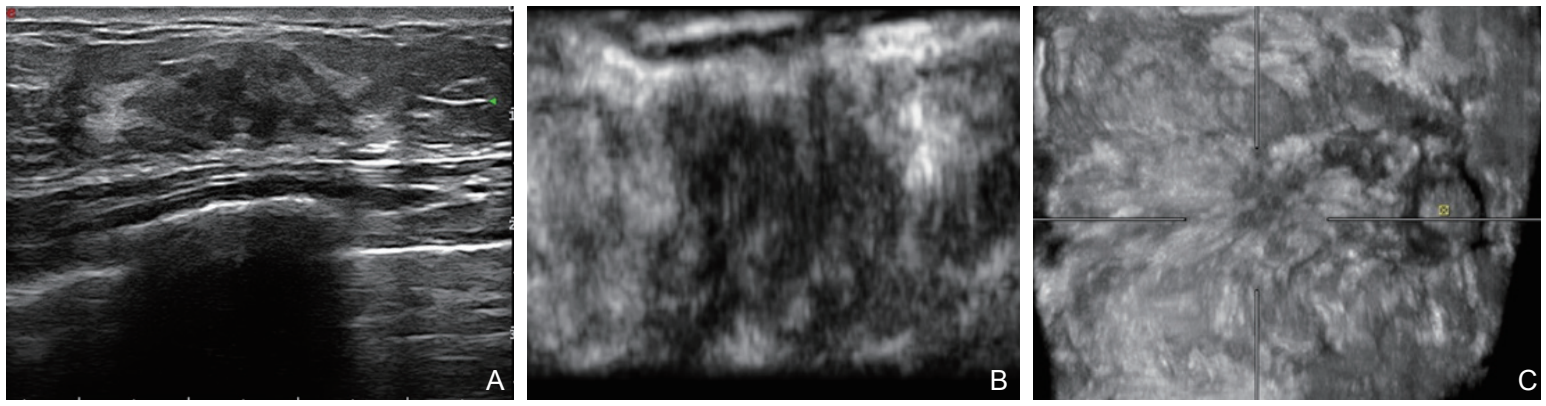

Figure 1 A 33-year old woman with invasive ductal carcinoma in the right breast. (A) Conventional ultrasound showed a hypoechoic lesion (11.2 $\times$ $10.1 \mathrm{~mm}$ ) with an oval shape and indistinct margin, and the final category was BI-RADS 4B for US; (B) Based on the findings in 3D-US imagings, the final BI-RADS category was BI-RADS 4B; (C) ABVS images showed the retraction phenomenon in the coronal plane, the final category was increased to BIRADS 4C. BI-RADS, breast imaging reporting and data system; US, ultrasound; 3D-US, three-dimensional US; ABVS, automated breast volume scanner.
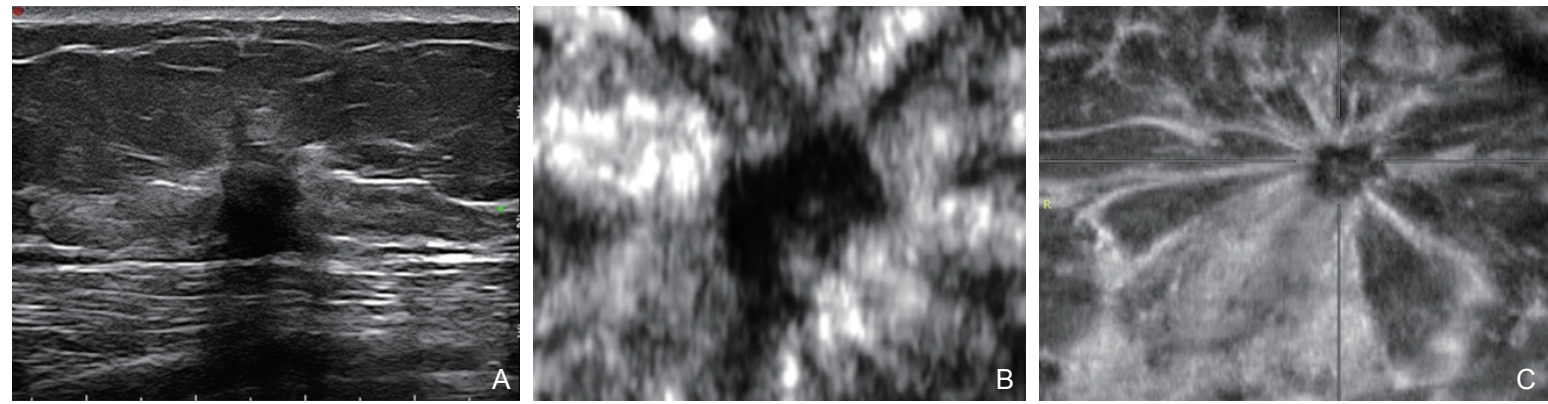

Figure 2 A 62-year old woman with invasive ductal carcinoma in the right breast. (A) Conventional ultrasound showed a hypoechoic lesion (15.5 $\times 10.5 \mathrm{~mm}$ ) with an irregular shape, spiculated margin, and posterior shadowing, and the final category was BI-RADS 4C for US. (B) 3D-US images displayed the retraction phenomenon in the coronal plane, the final BI-RADS category remained as BI-RADS 4C; (C) ABVS images showed the retraction phenomenon in the coronal plane, the final category was BI-RADS 4C. BI-RADS, breast imaging reporting and data system; US, ultrasound; 3D-US, threedimensional US; ABVS, automated breast volume scanner.
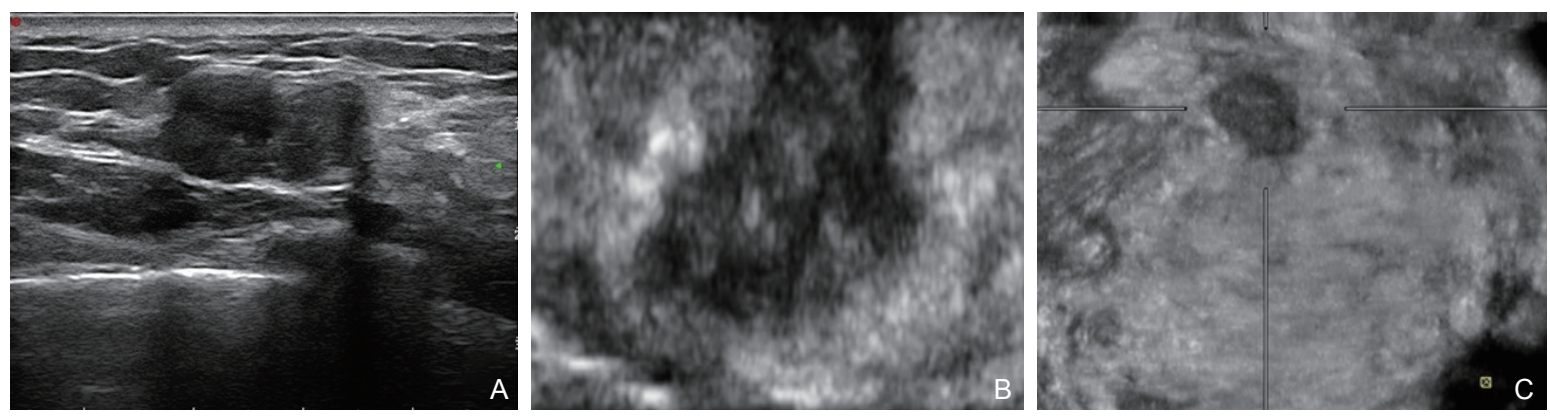

Figure 3 A 37-year old woman with intraductal papilloma in the left breast. (A) Conventional ultrasound showed a hypoechoic lesion (17.5 $\times 11.0 \mathrm{~mm})$ with irregular shape and circumscribed margin and the final category was BI-RADS 4A. (B) Based on the findings in 3D-US imagings, the final BI-RADS category was BI-RADS 4B. (C) ABVS images did not show the retraction phenomenon in the coronal plane, the final category was BI-RADS 4A. BIRADS, breast imaging reporting and data system; 3D-US, three-dimensional US; US, ultrasound; ABVS, automated breast volume scanner. 
Comparison of retraction phenomenon between $3 D$ US and $A B V S$

Using 3D-US, 31 breast lesions showed the retraction phenomenon (34.44\%), among which 22 lesions were proven to be malignant. Simultaneously, 25 lesions showed this phenomenon by ABVS (27.78\%), and 23 cases were confirmed as malignant. For 3D-US, the sensitivity, specificity, and area under the curve (AUC) were $62.86 \%, 83.64 \%$, and 0.732 , respectively, while for ABVS, they were $65.71 \%, 96.36 \%$, and 0.810 , respectively. The AUC of ABVS was higher than that of 3D-US, but no statistical significance was found between them $(P>0.05)$ (Fig. 1 and 2). Specifically, of the 10 lesions that showed the retraction phenomenon in 3D-US but not in ABVS, 7 cases were confirmed to be benign, while 3 cases were malignant. In addition, all 4 lesions showed the phenomenon in ABVS but not in 3D-US were malignant (Table 3 ).

\section{Diagnostic performances of $U S, 3 D-U S$, and $A B V S$ individually}

The diagnostic performances of the final BI-RADS category by US, 3D-US, and ABVS were individually illustrated in Table 4. For conventional US, the final BIRADS category was classified as BI-RADS $3(n=18)$, BI-RADS 4A $(n=26)$, BI-RADS 4B $(n=11)$, BI-RADS $4 \mathrm{C}(n=15)$, and BI-RADS $5(n=20)$, with a sensitivity and specificity of $82.86 \%$ and $89.09 \%$, respectively. ABVS was classified as BI-RADS $3(n=14)$, BI-RADS 4A $(n=23)$, BI-RADS 4B $(n=15)$, BI-RADS 4C $(n=21)$, and BI-RADS $5(n=17)$, with a sensitivity and specificity of $80.00 \%$ and $81.82 \%$, respectively. For 3D-US, the sensitivity and specificity were $85.71 \%$ and $73.73 \%$ with BI-RADS $3(n=10)$, BI-RADS $4 \mathrm{~A}$ $(n=20)$, BI-RADS 4B $(n=15)$, BI-RADS 4C $(n=32)$, and BI-RADS $5(n=13)$. 3D-US exhibited the best sensitivity, with a value of $85.71 \%$, and conventional US had the highest specificity at $89.09 \%$. In addition, conventional US performed the best with an AUC of 0.913, which was higher than those of 3D-US (0.842) and ABVS (0.871). However, no statistical significance was found between US and 3D-US $(P=0.05)$ and US vs. ABVS $(P=0.21)$. The kappa value for the BI-RADS category was 0.793 for US and ABVS, 0.644 for US and 3D-US, and 0.663 for 3D-US and ABVS.

\section{Diagnostic performances of $U S$ in combination with $3 D-U S$ and $A B V S$}

The retraction phenomenon of $3 \mathrm{D}-\mathrm{US}$ and ABVS was used in the conventional US to modify the BIRADS category. The modified BI-RADS category was BI-RADS $3(n=17)$, BI-RADS 4A $(n=21)$, BI-RADS 4B $(n=16)$, BI-RADS 4C $(n=9)$, and BI-RADS 5 $(n=27)$ when combined with 3D-US. The diagnostic performance showed sensitivity and specificity of up to $85.71 \%$ and $89.09 \%$, respectively, in the US+3D-US group. Simultaneously, they were BI-RADS $3(n=17)$, BI-RADS 4A $(n=21)$, BI-RADS 4B $(n=14)$, BI-RADS $4 \mathrm{C}(n=8)$, and BI-RADS $5(n=30)$ when combined with ABVS. The sensitivity and specificity increased to $88.57 \%$ and $87.27 \%$, respectively, in the US+ABVS group. The AUC reached up to 0.919 and 0.924 for these two groups. Only the diagnostic performance of 3DUS was significantly improved when combined with US $(P=0.02)$. Although US+ABVS had the best diagnostic performance, no significant difference was found among conventional US, US+3D-US $(P=0.70)$, and US+ABVS $(P=0.52)$ (Table 5, Fig. 4).

Table 3 Retraction phenomenon in 3D-US and ABVS

\begin{tabular}{lcc}
\hline Item & 3D-US & ABVS \\
\hline Retraction phenomenon & 31 & \\
Presence & 59 & 65 \\
Absence & & \\
Diagnostic performance & 62.86 & 65.71 \\
Sensitivity & 83.64 & 96.36 \\
Specificity & 0.732 & 0.810 \\
\hline AUC &
\end{tabular}

3D-US, three-dimensional ultrasound; ABVS, automated breast volume scanner; AUC, area under the curve

Table 4 Diagnostic performances of US, 3D-US, and ABVS

\begin{tabular}{|c|c|c|c|c|c|c|c|c|}
\hline \multirow{2}{*}{ Item } & \multicolumn{5}{|c|}{ BI-RADS category } & \multicolumn{3}{|c|}{ Diagnostic performances } \\
\hline & 3 & $4 \mathrm{~A}$ & 4B & $4 \mathrm{C}$ & 5 & Sensitivity & Specificity & AUC \\
\hline US & 18 & 26 & 11 & 15 & 20 & 82.86 & 89.09 & $0.913(0.835-0.962)$ \\
\hline 3D-US & 10 & 20 & 15 & 32 & 13 & 85.71 & 72.73 & $0.842(0.749-0.910)$ \\
\hline ABVS & 14 & 23 & 15 & 21 & 17 & 80.00 & 81.82 & $0.871(0.784-0.933)$ \\
\hline
\end{tabular}

US, ultrasound; 3D-US, three-dimensional US; ABVS, automated breast volume scanner; BI-RADS, breast imaging reporting and data system; AUC, area under the curve 
Table 5 Diagnostic performances of US in combination with 3D-US and ABVS

\begin{tabular}{|c|c|c|c|c|c|c|c|c|}
\hline \multirow{2}{*}{ Item } & \multicolumn{5}{|c|}{ Modified BI-RADS category } & \multicolumn{3}{|c|}{ Diagnostic axperformances } \\
\hline & 3 & $4 \mathrm{~A}$ & 4B & $4 \mathrm{C}$ & 5 & Sensitivity & Specificity & AUC \\
\hline US+3D-US & 17 & 21 & 16 & 9 & 27 & 85.71 & 89.09 & $0.919(0.842-0.966)$ \\
\hline US+ABVS & 17 & 21 & 14 & 8 & 30 & 88.57 & 87.27 & $0.924(0.848-0.969)$ \\
\hline
\end{tabular}

US, ultrasound; 3D-US, three-dimensional US; ABVS, automated breast volume scanner; BI-RADS, breast imaging reporting and data system; AUC, area under the curve

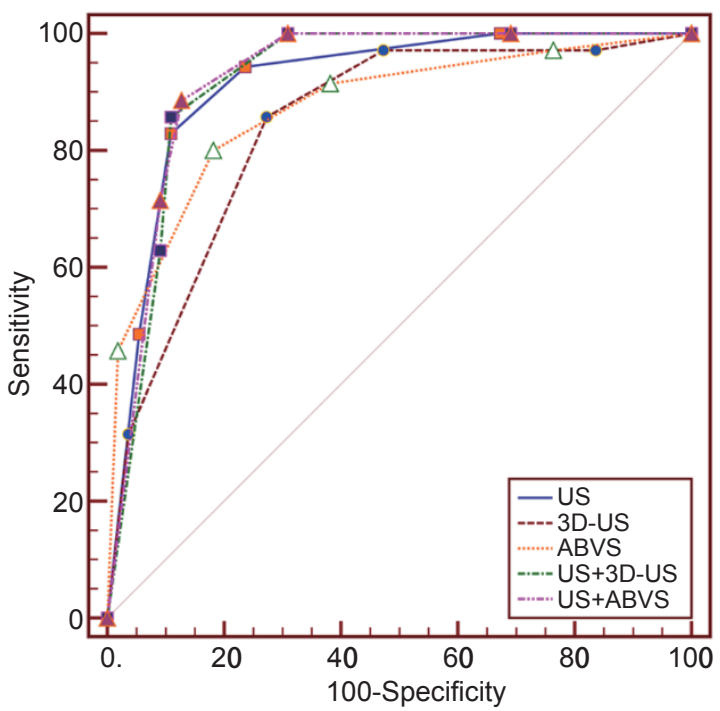

Figure 4 The receiver operating curves (ROC) of conventional US, 3DUS, ABVS individually and 3D-US combined with US, ABVS combined with US. US, ultrasound; 3D-US, three-dimensional US; ABVS, automated breast volume scanner.

\section{Discussion}

This study was carried out to compare 3D-US and ABVS in terms of breast lesion characterization, which, to the best of our knowledge, has never been reported previously.

Although the principles and operational systems are different between 3D-US and ABVS, one advantage of these two techniques over the conventional US lies in the multiplanar reconstruction of images, including sagittal, transverse, and coronal planes, which can clearly show the anatomical details and spatial location of the breast lesions, and thus improve the overall diagnostic performance [11].

The image quality of the US should be guaranteed for the characterization of breast lesions. A previous study showed that the image quality of ABVS was identical or superior to that of conventional US in $97.1 \%$ of breast cases [29]. Our preliminary results suggested that the overall image quality in the coronal plane was similar for most breast lesions between ABVS and 3D-US, while ABVS was superior to 3D-US in displaying adjacent structures of the lesion. The reason may be the small field of view for 3DUS [14,30], while the ABVS images consisted of a $15.4 \mathrm{~cm}$ $\times 16.8 \mathrm{~cm}$ field of view of the breast.
The diagnostic performances of individual US, 3DUS, and ABVS were compared in our study. In a recent meta-analysis of nine studies, the pooled sensitivity, specificity, and AUC were $90.8 \%$ (95\% confidence interval: $88.3-93.0 \%), 82.2 \%(80.0-84.2 \%)$, and 0.93 for ABVS, respectively, and 90.6\% (88.1 - 92.8\%), $81.0 \%$ (78.8 - 83.0\%), and 0.94 for conventional US by Wang et al. [15]. In addition, for 3D-US, the pooled sensitivity and specificity were calculated to be 0.923 (95\% confidence interval, $0.896-0.945$ ) and 0.872 (95\% CI, $0.849-0.893$ ), which was reported by Lian et al. [22]. The overall diagnostic performance of these three imaging techniques was not as good as described previously, which may be due to the small sample size and selective bias; as for the similar diagnostic capability between 3D imaging and the conventional US, the results conformed to those of previous studies [14,20,31-34]. Compared with 3D-US and ABVS, the BI-RADS category of conventional US performed the best with an AUC value of 0.913 , and it was 0.871 for ABVS and 0.842 for $3 \mathrm{D}-\mathrm{US}$, and the agreement between US and ABVS was considered as good, in accordance with Girometti et al. [16,24]. At this point, it seemed that a single ABVS could outperform 3D-US; however, no significant difference could be found among them.

The retraction phenomenon in the coronal plane has been reported as a significant and discriminating sign in the differential diagnosis of breast lesions $[10,35,36]$. Zheng et al. found it to be the best predictor of malignant breast lesions [37]. In our study, the retraction phenomenon differed significantly between benign and malignant breast lesions for both 3D-US and ABVS (Fig. 1 and 2), and the sensitivity and specificity were $65.71 \%$ and $96.39 \%$ for ABVS, which conformed to the previous literature, with sensitivities ranging from $57.5 \%$ to $91.4 \%$ and specificity from $92.5 \%$ to $100 \%$ [35,37-39]. For 3D-US, the sensitivity and specificity were $62.86 \%$ and $83.64 \%$, respectively. All four lesions that displayed retraction phenomenon in ABVS but not in 3D-US were confirmed to be malignant (Fig. 1), which indicated that ABVS seemed to be better than 3D-US for this sign, but no statistical significance was found between them. These results indicated that both $3 \mathrm{D}-\mathrm{US}$ and ABVS 
enabled better visualization of the lesion extent and infiltration degree and provided relatively high diagnostic performance.

Besides, the special retraction phenomenon, which was unique to the coronal plane, has been recommended for BI-RADS-US lexicon as an independent descriptor by Zheng et al. [37]. Therefore, the retraction phenomenon in 3D-US and ABVS was further combined to the conventional US for a modified BI-RADS in our study, and the diagnostic efficiency was greatly improved, among which the AUC for 3D-US and ABVS reached up to 0.919 and 0.924 with a significant difference from single 3D-US but not from the conventional US. The results were inspiring because combination could make the best of the advantage of the conventional US and the coronal plane information provided by $3 \mathrm{D}$ US and ABVS. On the other hand, it could avoid the shortcomings of ABVS, which could not provide the vascular and elastic characteristics of breast lesions.

Our study had some limitations. First, there might be a selection bias because the population included women with abnormal findings on imaging, with palpable masses or nipple discharge, but not the screening populations. Second, the analysis based on lesion size, histological types and molecular subtypes were not carried out in our study, which may also affect the results, since ABVS was demonstrated to be better at small or intraductal lesions $[24,25]$. Third, the sample size was small, and further studies would be needed to confirm this conclusion.

\section{Conclusion}

ABVS was superior in displaying the adjacent structure of the breast lesions. Moreover, compared with 3D-US, ABVS seems to be superior in showing the retraction phenomenon and in the characterization of breast lesions by itself or in combination with conventional US. Although no significant difference can be found between them, both ABVS and 3D-US provided valuable information in the coronal plane and improved our confidence in breast lesion characterization, especially when combined with the conventional US.

\section{Acknowledgements}

This study was supported by the the National Natural Science Foundation of China (grant no. 82071928).

\section{Conflict of Interest}

The authors have no conflict of interest to declare.

\section{References}

[1] Bae MS, Moon WK, Chang JM, Koo HR, Kim WH, Cho N, et al.
Breast cancer detected with screening US: reasons for nondetection at mammography. Radiology 2014; 270: 369-377.

[2] Yoo KB, Kwon JA, Cho E, Kang MH, Nam JM, Choi KS, et al. Is mammography for breast cancer screening cost-effective in both Western and asian countries?: results of a systematic review. Asian Pac J Cancer Prev 2013; 14: 4141-4149.

[3] Mandelson MT, Oestreicher N, Porter PL, White D, Finder CA, Taplin $\mathrm{SH}$, et al. Breast density as a predictor of mammographic detection: comparison of interval- and screen-detected cancers. J Natl Cancer Inst 2000; 92: 1081-1087.

[4] Harvey JA, Bovbjerg VE. Quantitative assessment of mammographic breast density: relationship with breast cancer risk. Radiology 2004; 230: 29-41.

[5] Berg WA, Zhang Z, Lehrer D, Jong RA, Pisano ED, Barr RG, et al. Detection of breast cancer with addition of annual screening ultrasound or a single screening MRI to mammography in women with elevated breast cancer risk. JAMA 2012; 307: 1394-1404.

[6] Uchida K, Yamashita A, Kawase K, Kamiya K. Screening ultrasonography revealed $15 \%$ of mammographically occult breast cancers. Breast cancer 2008; 15: 165-168.

[7] Hooley RJ, Greenberg KL, Stackhouse RM, Geisel JL, Butler RS, Philpotts LE. Screening US in patients with mammographically dense breasts: initial experience with Connecticut Public Act 09-41. Radiology 2012; 265: 59-69.

[8] Kolb TM, Lichy J, Newhouse JH. Comparison of the performance of screening mammography, physical examination, and breast US and evaluation of factors that influence them: an analysis of 27,825 patient evaluations. Radiology 2002; 225: 165-175.

[9] Jiang J, Chen YQ, Xu YZ, Chen ML, Zhu YK, Guan WB, et al. Correlation between three-dimensional ultrasound features and pathological prognostic factors in breast cancer. Eur Radiol 2014; 24 : 1186-1196.

[10] Watermann DO, Foldi M, Hanjalic-Beck A, Hasenburg A, Lughausen A, Prompeler $\mathrm{H}$, et al. Three-dimensional ultrasound for the assessment of breast lesions. Ultrasound Obstet Gynecol 2005; 25: 592-598.

[11] Guo R, Lu G, Qin B, Fei B. Ultrasound imaging technologies for breast cancer detection and management: a review. Ultrasound in medicine \& biology 2018; 44: 37-70.

[12] Meyberg-Solomayer GC, Kraemer B, Bergmann A, Kraemer E, Krainick U, Wallwiener D, et al. Does 3-D sonography bring any advantage to noninvasive breast diagnostics? Ultrasound Med Biol 2004; 30: 583-589.

[13] Clauser P, Londero V, Como G, Girometti R, Bazzocchi M, Zuiani C. Comparison between different imaging techniques in the evaluation of malignant breast lesions: can 3D ultrasound be useful? Radiol Med 2014; 119: 240-248.

[14] Kotsianos-Hermle D, Hiltawsky KM, Wirth S, Fischer T, Friese K, Reiser M. Analysis of 107 breast lesions with automated 3D ultrasound and comparison with mammography and manual ultrasound. Eur J Radiol 2009; 71: 109-115.

[15] Wang L, Qi ZH. Automatic breast volume scanner versus handheld ultrasound in differentiation of benign and malignant breast lesions: a systematic review and meta-analysis. Ultrasound Med Biol 2019; 45: 1874-1881.

[16] Vourtsis A, Kachulis A. The performance of 3D ABUS versus HHUS in the visualisation and BI-RADS characterisation of breast lesions in a large cohort of 1,886 women. Eur Radiol 2018; 28: 592-601.

[17] Yang S, Gao X, Liu L, Shu R, Yan J, Zhang G, et al. Performance and reading time of automated breast US with or without computeraided detection. Radiology 2019; 292: 540-549.

[18] van Egdom LSE, Lagendijk M, Heijkoop EHM, Koning AHJ, van 
Deurzen CHM, Jager A, et al. Three-dimensional ultrasonography of the breast; An adequate replacement for MRI in neoadjuvant chemotherapy tumour response evaluation? - RESPONDER trial. Eur J Radiol 2018; 104: 94-100.

[19] Tozaki M, Isobe S, Yamaguchi M, Ogawa Y, Kohara M, Joo C, et al. Optimal scanning technique to cover the whole breast using an automated breast volume scanner. Jpn J Radiol 2010; 28: 325-328.

[20] Cho KR, Seo BK, Lee JY, Pisano ED, Je BK, Lee JY, et al. A comparative study of 2D and 3D ultrasonography for evaluation of solid breast masses. Eur J Radiol 2005; 54: 365-370.

[21] Cho N, Moon WK, Cha JH, Kim SM, Han BK, Kim EK, et al. Differentiating benign from malignant solid breast masses: comparison of two-dimensional and three-dimensional US. Radiology 2006; 240: 26-32.

[22] Bin L, Huihui Y, Weiping Y, Changyuan W, Qinghong Q, Weiyu M. Value of three-dimensional ultrasound in differentiating malignant from benign breast tumors: a systematic review and meta-analysis. Ultrasound Q 2019; 35: 68-73.

[23] Kim H, Cha JH, Oh HY, Kim HH, Shin HJ, Chae EY. Comparison of conventional and automated breast volume ultrasound in the description and characterization of solid breast masses based on BIRADS features. Breast cancer 2014; 21: 423-428.

[24] Girometti R, Zanotel M, Londero V, Linda A, Lorenzon M, Zuiani C. Automated breast volume scanner (ABVS) in assessing breast cancer size: a comparison with conventional ultrasound and magnetic resonance imaging. Eur Radiol 2018; 28: 1000-1008.

[25] Choi EJ, Choi H, Park EH, Song JS, Youk JH. Evaluation of an automated breast volume scanner according to the fifth edition of BIRADS for breast ultrasound compared with hand-held ultrasound. Eur J Radiol 2018; 99: 138-145.

[26] Girometti R, Zanotel M, Londero V, Bazzocchi M, Zuiani C. Comparison between automated breast volume scanner (ABVS) versus hand-held ultrasound as a second look procedure after magnetic resonance imaging. Eur Radiol 2017; 27: 3767-3775.

[27] Mercado CL. BI-RADS update. Radiol Clin North Am 2014; 52: 481-487.

[28] Li Z, Tian J, Wang X, Wang Y, Wang Z, Zhang L, Jing H, et al. Differences in multi-modal ultrasound imaging between triple negative and non-triple negative breast cancer. Ultrasound Med Biol 2016; 42: 882-890.
[29] An YY, Kim SH, Kang BJ. The image quality and lesion characterization of breast using automated whole-breast ultrasound: A comparison with handheld ultrasound. Eur J Radiol 2015; 84: 1232-1235

[30] Chen L, Chen Y, Diao XH, Fang L, Pang Y, Cheng AQ, et al. Comparative study of automated breast 3-D ultrasound and handheld B-mode ultrasound for differentiation of benign and malignant breast masses. Ultrasound Med Biol 2013; 39: 1735-1742.

[31] Wang HY, Jiang YX, Zhu QL, Zhang J, Dai Q, Liu H, et al. Differentiation of benign and malignant breast lesions: a comparison between automatically generated breast volume scans and handheld ultrasound examinations. Eur J Radiol 2012; 81: 3190-3200.

[32] Wang ZL, Xu JH, Li JL, Huang Y, Tang J. Comparison of automated breast volume scanning to hand-held ultrasound and mammography. Radiol Med 2012; 117: 1287-1293.

[33] Kim SH, Kang BJ, Choi BG, Choi JJ, Lee JH, Song BJ, et al. Radiologists' performance for detecting lesions and the interobserver variability of automated whole breast ultrasound. Korean J Radiol 2013; 14: 154-163.

[34] Schmachtenberg C, Fischer T, Hamm B, Bick U. Diagnostic performance of automated breast volume scanning (ABVS) compared to handheld ultrasonography with breast mri as the gold standard. Acad Radiol 2017; 24: 954-961.

[35] Zanotel M, Bednarova I, Londero V, Linda A, Lorenzon M, Girometti $\mathrm{R}$, et al. Automated breast ultrasound: basic principles and emerging clinical applications. La Radiologia medica 2018; 123: 1-12.

[36] Rotten D, Levaillant JM, Zerat L. Analysis of normal breast tissue and of solid breast masses using three-dimensional ultrasound mammography. Ultrasound Obstet Gynecol 1999; 14: 114-124.

[37] Zheng FY, Yan LX, Huang BJ, Xia HS, Wang X, Lu Q, et al. Comparison of retraction phenomenon and BI-RADS-US descriptors in differentiating benign and malignant breast masses using an automated breast volume scanner. Eur J Radiol 2015; 84: 2123-2129.

[38] Lin X, Wang J, Han F, Fu J, Li A. Analysis of eighty-one cases with breast lesions using automated breast volume scanner and comparison with handheld ultrasound. Eur J Radiol 2012; 81: 873-878.

[39] Xiao YM, Chen ZH, Zhou QC, Wang Z. The efficacy of automated breast volume scanning over conventional ultrasonography among patients with breast lesions. Int J Gynaecol Obstet 2015; 131: 293296. 